\title{
INTERACTIVE NARRATION IN THE DIGITAL AGE
}

\author{
Huang Minfen
}

Chinese Department, Xiamen University, Xiamen, 361005

\begin{abstract}
Interactive narration based on new media has become important research topic for postnarratology. It features non-linearity and can be classified as person-to-person, person-to-machine, and machine-to-machine. In order to fulfil the potential of interactive narration, we have to deal with the contradiction between narration and interaction properly, and lucubrate the motive of interactors and the narrative effects on them.
\end{abstract}

Keywords: interaction, narration, digital art

\section{摘要}

基于新媒体的交互性叙事是后经典叙事学的重要研究课题。它以非线性为特征, 具备人际交

互、人机交互、机机交互等类型。为了充分发挥交互性叙事的潜能，必须妥善处理叙事与交互 性的矛盾, 深入研究交互动机、交互效果等问题。

关键词: 叙事 交互 新媒体 


\section{前言}

交互性叙事在人类历史上堪称源远流长。在口头传播占主导地位的时代, 交互性叙事就获得了 广泛的应用, 原因主要是口语本身支持对话。由于文字的发明、印刷术的问世, 书面传播后来 居上，限制了交互性叙事的发展空间。这种状况在交互性电子媒体起之后才渐渐改变。如今 “交互性”已经成为口耳相传的术语，交互性叙事也在新技术基础上放射出前所未有的异彩。

\section{（一）交互性叙事的理念}

有关叙事的探讨由来已久。柏拉图对模仿（mimesis）和叙事（diegesis）的区分被认为 是西方哲人相关探讨的发端。其后, 人们对于叙事的研究深入到叙事视点、叙事声音、叙事距 离等方面。尽管如此, 叙事学到 20 世纪才发展成为一门学科。俄国形式主义者 (尤其是普洛 普) 为它的奠基做出了重要贡献。从词源的角度看, 叙事学 (narratologie) 一词是法国学者托 多罗夫（Tzvetan Todorov）发明的（1969）， (Todorov, Tzvetan. Grammaire du Décaméron. The Hague: Mouton, 1969:69) 由拉丁文词根 narrato（叙述）和希腊文词尾 logie（科学）组合而成。 它的英语对应词为 narratology。托罗多夫将叙事学定义为关于叙事结构的理论, 采用先将叙事 现象分解成若干组件、再确定其功能和相互关系的研究方法。换言之, 经典叙事学重视文本叙 事结构研究, 重在对叙事文本进行技术分析, 与结构主义有着密切关系。兴起于 20 世纪 60 年 代的后结构主义关注叙事的 “不完全性” （incompleteness）, 认可以读者为中心的叙事无穷演 化的可能性。后现代主义将线性叙事当成人类理性幻化的产物来加以反对, 因为客观世界并不 存在作为其基础的线性因果关系。经过 20 余年的发展, 西方叙事学研究范式到上个世纪末发 生重大变化, 其中之一就是由强调作者赋予叙事的意义向强调读者对于意义的阐释转变。这种 转变是经典叙事学转向后经典叙事学的标志之一。后经典叙事学不仅关注读者的阐释框架和阐 释策略, 而且关注文本自身的特征, 将读者与文本的相互作用当成自己的研究重点。就此而 言, 作为理论的后经典叙事学和作为实践的交互性叙事颇有相通之处。交互性叙事的经验丰富 了后经典叙事学，后经典叙事学应当能够对交互性叙事给予理论指导。

一般认为: 交互性叙事以非线性为特征。丹麦哥本哈根大学媒体研究教授戈柔窦（Torben Grodal）在 2003 年就此指出：术语 “非线性” 密切联系于交互性问题, 因为对人文科学领域的 许多学者来说, 完全交互性与 “最高作用” 这一术语相链接。这一术语被赋予由后现代主义一 解构主义思考的不同分支 (例如源于德里达的思考) 所提供的联系。根据他们的哲学, 线性是 西方形而上学的逻各斯思考 (即因果关系) 的产物, 为字母写作的线性所强化。因此, 我们可 以认为故事在其实现中本质上是线性的：1）所有的文本与经历作为体验过程都是线性的, 因 为即使在 “阅读” 超文本时经验流也总是线性的；2）故事作为有意义的事件的系列是线性 的, 因为有意义的故事依赖于因果关系、不可逆转的过程以及行动轨迹的选择。当然, 既定故 事世界可能支持不同故事、不同路径选择，以及计算机故事中不同玩家的表演）。然而，我们 必须区分故事体验及故事世界供给, 因为既定故事世界或游戏可能提供一种或若干故事体验 (Grodal, Torben.Stories for Eye, Ear, and Muscles: Video Games, Media, and Embodied Experiences. In The Video Game Theory Reader.Edited by Mark J. P. Wolf, and Bernard Perron. New York: Routledge, 2003:145-147)。从戈柔窦的论述中可以看出: “交互性叙事” 本身包含了悖论: 作 为 “叙事” , 它实际上倾向于线性; 作为 “交互性”, 它又倾向于非线性。西拉斯（Nicolas Szilas）曾经区分出设计交互性戏剧的三个主要问题：其一, 如何建构交互戏剧系统: 什么样 的叙事原则可以建构这类系统的基础？如何才可能与一个故事相互作用？如何克服故事与交互 性之间乍看起来的矛盾? 人工智能在这一任务中起什么作用? 其二, 用户的角色是什么：对用 户来说变成一个故事中的人物意味着什么? 她在故事中的叙事角色是什么? 她如何与系统相互 
作用? 什么样的现有体验（书籍、视频游戏、角色扮演、儿童玩要等）与交互性戏剧最为接 近? 其三，作者的角色是什么？用户如何通过交互性戏剧传达其意图与价值观？作者是否在交 互性戏剧中 “放弃控制” ? 在故事中的事件过程最终将掌握在用户手里的情况下，如何 “写一 个故事”？以上三个问题不是彼此独立的，第一个问题最为关键 (Szilas, Nicolas. Authoring Highly Generative Interactive Drama.In Virtual Storytelling: Using Virtual Reality Technologies for Storytelling. Second International Conference, ICVS 2003, Proceedings. Edited by Olivier Balet, Gé rard Subsol, and Patrice Torguet. Berlin: Springer, 2003:37-46)。上述问题对于交互性叙事是有普 遍意义的。为了准确把握各种不同作品的特点, 瑞安主张对以下四种情况加以区分（2005）: 一是外围交互性, 二是影响叙事话语和故事陈述的交互性, 三是在部分前定的故事中创造变化 的交互性, 四是实时故事生成交互性(Marie-Laure,Ryan.Peeling the Onion: Layers of Interactivity in Digital Narrative Texts.Based on a talk presented at the Conference "Interactivity of Digital Texts," Münster, Germany, May 2005)。

\section{（二）交互性叙事的类型}

数码时代的交互性叙事有三种可能的模式: 一是人际交互型，二是人机交互型，三是机 机交互型。在相当长的历史时期内, 交互性叙事以人际交互型为主, 机器仅仅是作为人际交互 的辅助手段起作用, 局限于灯光、音响、舞台、道具、机关布景等领域。新媒体使人际交互得 以用电子速度远程进行, 从而改变了人际交互的态势。由于计算机和网络的智能化, 人机交互 越来越引人瞩目, 成为交互性叙事发展的新动力。在以之为基础的交互性叙事中, 相当一部分 角色是由机器 (智能程序) 来扮演的。正如劳雷尔 (1991) 所指出的: 设计良好的基于系统的 人物可以对戏剧投入作出贡献, 激发神入, 影响加入同一活动的真人的行动与感情 (Laurel, Brenda. Computers as Theatre. Reading, Mass.: Addison-Wesley Publishing Company, 1991:146)。 不过，正如葡萄牙索布拉尔（Daniel Sobral）等人所指出的，交互性叙事中的人物经常与自动 代理的存在相联系（这些代理可以用相当自由的方式行动）, 问题是无法传达有目的的意义 (Sobral.Daniel, et al. Managing Authoring in Plot Conduction. In Virtual Storytelling: Using Virtual Reality Technologies for Storytelling. Second International Conference, ICVS 2003, Proceedings. Edited by Olivier Balet, Gérard Subsol, and Patrice Torguet. Berlin: Springer, 2003:57)。机机交互型 的叙事目前还处在实验阶段。

如果说以计算机为基础的交互性叙事有什么特点的话，其中之一就是它为人类用户创造 了变形的多种可能性。正如默里所指出的, 转换的愉悦是数码环境的特色之一。计算机为我们 提供了无数的变形方式。不仅我们自己可以在虚拟现实中由活人变形为高飞的鸟儿, 而且词 语、数字、图像都更为可塑, 都更有动感。计算机的变形力量在叙事环境中特别诱人。因为数 码对象可拥有多重例示, 它们自身就能引发我们对其变形的愉悦。在《真人快打》（Mortal Kombat）里，可从 16 个对手中任选两个开打，而且交互者可以任选一边。MUDs 就所支持的 角色的多样性展开竞争。在计算机游戏中, 我们作为玩家并非只有一条命 (甚至对我们的文明 来说也是如此）。如果不殹意的话, 可重新来过。不过, 如何利用变形来取得精妙的效果, 有 待探索。一个作者如何在如此易变的环境中讲述一个连贯的故事? 读者怎么知道孰为头尾? 我 们需要新的形式惯例以驾驭易变性。为此, 我们要弄清从变形文学中追求什么类型的愉悦 (Murray, Janet Horowitz. Hamlet on the Holodeck. New York, NY: The Free Press, 1997:154-155)。

在具体作品中, 交互性叙事的设计是多种多样的。保罗曾经谈到, “数码艺术中这种复 杂互动的可能性已经超出仅仅是提供观看艺术作品的熟练形式的 “点击” , 或者用户行动触发 特定反应的交互性。更为本质的变化伴随着虚拟艺术对象而发生, 这些对象是结尾开放的 “信 息叙事” , 有变动的结构、逻辑与结局, 其中, 对于内容、语境与时间的控制通过互动转移到 各自的受者。这类作品可以取无数形式, 对于其视觉外观有可变等级的控制, 由艺术家或受众 
进行。数码艺术并非总是合作性的（在这个单词的原义上），但经常是参与性的，依赖于多用 户输入。在某些艺术作品中, 观者在由艺术家所设定的参数内互动; 在其他作品中, 他们自行 设定其参数, 或者变成基于时间的实况表演的参与者。在某些个案中, 一个艺术作品的虚拟显 示最终由观者创造: 没有输入, 一个作品可能完全由黑屏组成。” (Paul, Christiane.Digital Art. New York: Thames \& Hunson (World of Art), 2003:67-68.) 上述见解无疑对我们有启发意义。

\section{（三）交互性叙事的动机}

“动机” (motive, motif) 一词使用广泛, 含义比较复杂。在艺术领域, 动机可能是指 作品中重现的片段、主题、结构模式等要素。对于经典叙事学来说, 这种重现往往具备象征意 义, 因此值得重视。在心理学领域, 动机往往是指行为的内驱力, 由需要转化而来。从这一角 度看, 动机可以进一步区分为创作者（或开发者）的动机、艺术人物的动机、鉴赏者（读者、 观众或交互者）的动机等。这些动机可能彼此交错。以另类实境游戏（alternate reality game） 为例。它将现实世界用为平台来展开交互性叙事, 经常涉及多重媒体与游戏要素, 以讲述可能 会受参与者的观念或行动影响的故事。人物是由游戏设计者（而非由人工智能）控制的。游戏 者直接与游戏中的人物互动, 迎接基于情节的挑战, 解开谜团, 在这一过程中经常需要进行社 区合作。所应用的媒体有电话、邮件和互联网等。有时, 谜语在网上传播, 而玩家需要组队在 现实世界中寻找解题线索。由此看来, 交互性叙事所涉及的动机是相当复杂的。尽管如此, 由 于交互性叙事将用户的参与作为自己的特色, 因此, 有关读者、观众或交互者的动机成为研究 的重点。

交互性叙事之所以可能，从技术上说自然要从所凭借的媒体去探索。尽管如此，我们不 能忽视隐藏在其后的心理因素。瑞安探讨了隐藏在交互性之后的动机问题, 即读者为何要提供 反馈。她在《作为虚拟现实的叙事: 文学与电子媒体中的沉浸与交互性》（2001）一书中指 出: 交互性文本是由用户输入提供燃料的机器。读者为什么想提供输入呢? 一是决定情节。这 类交互性的例子, 是读者被要求决定人物命运的多重选择文本……是改变对文本世界的视 点。这可能意味着打开新的窗口、追随另一条情节线、将焦点转移到不同人物, 听到不同说法 的事实, 或者进入新的私人世界。三是探索可能的领域。如果读者获得机会尝试所有的选项, 交互性的目的便不再是决定情节（因为所有的分支如今都是情节的一部分），而是获得对可能 性的交织的全面视野, 并对作者作为 “歧路花园” 的设计者的表现加以评价。四是使文本机器 保持运行。当读者的选择完全盲目时, 点击某个链接的目的与其说是别的还不如说只是想让更 多文本呈现在屏幕上……五是调用文件。这是由超文本帮助文件或万维网搜索引擎所赋予的交 互性。在这种类型的动机中, 文本被作为可被带到用户屏幕上并用于个人项目的资源集合来对 待。六是玩游戏、解决问题。这一范围无须解释。七是评价文本。在印刷文学中, 回顾、讨论 与更为简单的购买书籍的过程是一种交互机制, 职业或业余的读者通过它告诉作者自己想要什 么。互联网上的聊天群体与公告牌可以同样地被用于对情节的评论, 因此影响电视系列剧或在 线肥皇剧的作者(Ryan, Marie-Laure. Narrative as Virtual Reality: Immersion and Interactivity in Literature and Electronic Media. Baltimore and London: The Johns Hopkins University Press, 2001:211-212)。上述分析还是比较全面的。

\section{（四）交互性叙事的矛盾}

叙事与交互性的矛盾为交互性叙事所特有，牵涉到开发者如何处理鉴赏者所进行的情节 内交互的问题。交互性叙事通常必须提供连贯而诱人的故事（即情节），同时又必须为鉴赏者 的参与提供方便的条件。这两个要素之间是有矛盾的。早在 20 世纪 80 年代, 劳雷尔就意识到 交互性叙事的上述矛盾, 并呼吁将事先设计好的情节与交互性有机结合起来, 但人们对此还缺 乏应有的注意。默里因此认为: 或许这种系统的故事的开发有赖于文艺家与计算机科学家之间 
远比目前的水平更为密切的合作。当然, 这不是说在这方面没有任何进展。值得一提的是卡耐 基 - 梅隆大学由贝茨领导的 $\mathrm{Oz}$ 小组所做的工作。该组将自己开发的系统定位为实况戏剧游 戏, 在其中一个交互者被置于公交车站威胁性情境中。参加演出的学生即兴扮演由计算机所产 生的人物（这些人物是自主行动的），通过头盔得到导演的指示。导演虽不在台上，但始终切 近观察表演的过程, 并适时提示种种情节。在学生所扮演的人物中有一个被确定为交互者。故 事的高潮是这样的时刻: 给交互者一把枪, 让他做出选择, 要么用枪对付恶棍以保护一个盲 人, 要么跳上正要开行的公共汽车逃之夭天。对于旁观者来说, 故事进展慢得可以, 而且高潮 到来时简直是乱成一团。为演出拍摄录像的人就这样认为。但是, 对于在剧中扮演交互者的学 生来说, 情节本身很生动, 而且, 当他拿到枪时, 深深体会到了作出道德选择的艰难一一毕竟 是实况演出啊。 $\mathrm{Oz}$ 小组所作的工作告诉我们: 就情节令人满意与否而言, 交互情境完全不同 于观众情境。为了保证演出有条不紊地进行，必须开发故事控制者（story controllers），以监 测环境中的所有要素，像棋手在复杂的策略中做出选择那样精确而有预见性地调整虚拟世界。 复杂的模式控制因运用计算机而成为可能。它正将故事推进到高度抽象而又多变的领域。但 是, 在追求复杂性、抽象性的时候, 面临着丧失连贯的危险。情节的任何抽象描写的成功, 取 决于在人类作家手中还保留有多少控制权。因此, 计算机化的抽象越少, 故事越是令人满意。 或者说: 迄今为止叙事模式的开发, 主要是认知科学家与计算机科学家合作的结果。我们必须 发现新的、与作家创作方式更为相近的抽象模式(Murray, Janet Horowitz. Hamlet on the Holodeck. New York, NY: The Free Press, 1997:202-203)。

索布拉尔 (Daniel Sobral) 等人《情节引导中的写作管理》（2003）一文进一步指出：为 了使叙事有交互性, 我们需要弹性, 以叙事流允许用户影响故事本身的方式。另一方面, 故事 作者需要保持某种结构、某种前定的叙事流, 以保证故事的高潮。这两个明显对立的目标导致 了叙事悖论, 即一再出现的陷阱, 多数交互性叙事环境 (interactive narrative environment, INE）无法避免。而且, 当我们将交互性叙事环境用于教育目标时, 新的要求暗示着对创造此 类系统的额外负担 (例如, 要保证特定事件为教学的理由发生)。以情节为中心的交互性叙事 通常与写成的脚本相联系。“即使是由人写成的情节对于叙事的引导来说是关键性的, 为切换 所必需参数数量可能在为这些基于情节的系统建构交互性时带来严重的问题。而且, 多数基于 情节的叙事系统将故事分成片断, 运用外在链接联系它们。这将巨大的努力强加给作者。不 过, 虽然做了某些使外部链接最少化甚至为零的努力, 这类企图离减轻经常强加给作者的无法 管理的写作困境还相差甚远。” 为了平衡这两个经常对立的目标, 交互性叙事系统必须支持弹 性写作过程, 将作为多数交互性 (非线性) 叙事写作之特征的按指数增长的工作（经常伴随分 叉结构而来。引者注）最小化。作者不应直接控制故事中人物的行为。相反，只应提供让智能 代理可以扮演其角色的背景。这一点也是为了将创作者（作家与设计者）和程序员之间的冲突 最小化。虽然有了奔向创作者懂编程的潮流以及对艺术家友好的编程语言, 现今项目的复杂性 仍要求不断增长的团队和更大的管理资源。这经常引发两种对立的角色之间的冲突，这种冲突 会降低双方角色在最终产品中的相关性, 而这种相关性毕竟最终是由程序员来执行的 (Sobral Daniel, et al. Managing Authoring in Plot Conduction. In Virtual Storytelling: Using Virtual Reality Technologies for Storytelling. Second International Conference, ICVS 2003, Proceedings. Edited by Olivier Balet, Gérard Subsol, and Patrice Torguet. Berlin: Springer, 2003:57-58)。

\section{（五）交互性叙事的效果}

交互性叙事的效果也为研究者所关心。默里认为: 本能的唤起不可过度。恐怖片如果太 过恐怖, 人们便会从影院跑出来; 爱情片如果太过撩逗, 观众便可能将视线从银幕移开。因 此, 对参与者的唤起必须细心加以调节。传统叙事对此是有所考虑的, 因此, 它们将唤起控制 在一定范围内, 不使接受者感到不快。计算机为参与性的创造提供了新舞台。我们逐渐学会了 演员所做之事, 并从情感上为明知不 “真” 的真实体验立法。数码空间的感觉呈现越是有说服 
力, 我们越是觉得自己出现在虚拟世界中, 并追求在那儿表演更大范围的行动。MUDs 与实况 角色扮演游戏 (Live Action Role Playing, LARP) 的玩家取之与挥之人格之易, 令人联想到在 扮演中经受训练的观众正在成长起来。我们正在变成世界剧目公司的一部分, 可以在更为复杂 的参与性故事中承担更为复杂的角色。我们正在一点点地发现将建构虚拟戏剧的第四堵墙的参 与常规, 以及将加深与保存沉浸感的魔力的表现性身势(Murray, Janet Horowitz. Hamlet on the Holodeck. New York, NY: The Free Press, 1997:120,125)。

某些人对于交互性叙事持肯定态度，其原因大致可以归纳为以下几方面：一是交互性叙 事顺应了艺术领域民主化的要求。斯托拉布拉斯认为：原则上，交互性提供了巨大的文化的、 社会的利益。它应该赋权给用户、鼓励文化活动（而非仅仅是旁观），使艺术对受众更有反应 性, 将艺术的排外的、（多数是）胁迫人的空间与话语向兼容性、民主性的微风开放 (Stallabrass,Julian. The Online Clash of Culture and Commerce. Millbank, London: Tate Publshing, p.68)。二是交互性叙事体现了数字复制时代特有的价值。莫尔指出： “在数字可复制时代，文 化的表现方式也经历了一种重要的转型。在独一无二的作品时代, 膜拜价值（cult value）构成 了作品的价值, 在机械复制时代, 展示价值（exhibition value）构成了作品的价值; 而在数字 复制时代, 则是操控价值 (manipulation value) 构成了再现的价值。” (莫尔《赛博空间的奥 德赛》, 麦永雄译, 广西师范大学出版社 2007 年版, 第 99 页)。三是交互性叙事符合当今用户 的心理要求。正因为如此, 美国宾夕法尼亚大学西尔弗曼（Barry G. Silverman）等人相信: 通 过以一种连贯的、可访问的格式向教育家团队提供娱乐体裁的技术进步, 开发交互式戏剧生成 器, 我们相信教育游戏的充分潜能可以实现(Silverman, Barry G. Authoring Edutainment Stories for Online Players (AESOP): Introducing Gameplay into Interactive Dramas. In Virtual Storytelling: Using Virtual Reality Technologies for Storytelling. Second International Conference, ICVS 2003, Proceedings. Edited by Olivier Balet, Gérard Subsol, and Patrice Torguet. Berlin: Springer, 2003:6572)。

另一方面，对于文学、戏剧与影视叙事交互化，都有人不以为然，有关论争不绝如缕。 根据记载, 1776 年有个叫西米尔・约翰逊（Simuel Johnson）的人宣称: “没有古怪的东西可 以持久。《项狄传》好景不长。” (Douglas, J. Yellowlees. The End of Books- - Or Books without End? Reading Interactive Narratives Ann Arbor: The University of Michigan Press, 2000, vii.) 由此看来, 当时交互性故事不见得为时人所看好。即使在近两个世纪之后, 这类作品仍有争 议。美国著名美学家苏珊・朗格（Suzanne Lenglen）曾援引自己的亲身经历，说明演员与观众 之间的直接交互破坏了戏剧幻象，所引发的异乎寻常的混乱使台上的演出无法进行下去。她指 出: “这种戏剧作品, 以及它所体现的戏剧概念的根本失误, 就在于完全忽略了爱德华-布洛 (Edward Bullough) 在其享有盛誉的论文中所提出的 “心理距离” 这一概念。所有的艺术欣赏 ——绘画、建筑、舞蹈, 不管哪种艺术都要求一定的超然态度, 显然, 这就是所谓的 “凝神观 照”, “审美态度” , 或欣赏者的 “客观性”。” (苏珊 - 朗格《情感与形式》, 刘大基等 译，中国社会科学出版社 1986 年版，第 368 页。布洛的论文题为《作为艺术因素和一种审美 原则的 “心理距离” 说》.发表于《英国心理学学》报 1912 年 6 月号。) [英国作家阿尔迪斯

（Brian Wilson Aldiss） 《审判》、美国作家布拉德伯里（Ray Bradbury） 《华氏温度计 451》 等科幻小说将交互性电视当成有利于社会监控和专制统治的媒体恶梦来描写。作为交互性叙事 之代表的游戏，受到了更为强烈的挞伐。罗斯扎克（Theodore Roszak）《信息崇拜一一计算机 神话与真正的思维艺术》（1986）一书认为: “即使在供儿童玩要的最简单的电子游戏里, 我 们也可以发现一些颇可置疑的设计思想。游戏里显然充斥着冷酷的竞争和疯狂的毁灭行为。取 胜是重要的, 杀戮受到推崇。” (罗斯扎克《信息崇拜一一计算机神话与真正的思维艺术》, 苗华健、陈体仁译，中国对外翻译出版公司 1994 版，第 108 页。) 
莫尔斯（Margaret Morse）则对交互性的所谓 “解放作用” 表示怀疑。她认为: 一旦 “活 性” 的同时性变成图像与世界之间的即刻反馈，一种倒置就发生在一度被称为呈现的东西之 中: 图像与世界都不是 “第一位” , 一个都像是在塑造另一个。“交互性” 被理解为一种允许 用户与观者在电话、键盘、远程控制、控制杆、鼠标、触摸屏、脑波阅读器等输入设备的帮助 之下选择或改变图像的手段。这样的交互性已经被错当成一种将改变交流之性质的解放性的自 我表达。例如, 双向电视被吹捧成为一种逃避为消费电视的单向与惰性的沙发位置。然而, 如 果交互性是一种对输入的显露的即时反馈的观念的拓展的话, 也就是说, 如果它是操作性的、 工具性的, 任何类型的输入手段都使得在电视上或在计算机显示器上的东西更为交互主体的或 解放性的?” (Morse, Margaret. Virtualities: Television, Media Art, and Cyberculture. Bloomington and Indianapolis: Indiana University Press, 1998:21-22.)

事实上, 简单地肯定或否定交互性叙事都是难以服人的。事实上, 简单地肯定或否定交 互性叙事都是难以服人的。默里对此有所体会。她举例说: 在玩游戏《西蒙城》（SimCity） 时, 人们可以体验到上帝般的快乐。玩家所充当的市长比现实生活中的任何政治领袖都更有权 力, 可以对资源施加无所不能的控制, 同时也对自己的行为的后果具有无所不在的意识。当 然, 游戏本身对于多种风格的玩家的开发性, 使得玩家有条件扮演不同类型的上帝。这两种因 素大大增加了游戏本身所带来的愉悦。在《锡德 - 米埃尔的文明》（Sid Meier'Civilization, 中 文版称为 “文明” 或 “文明帝国” ) 中，上述两种因素都得到了加强。玩家居然可以扮演某一 文明的领袖, 在许多世纪中发挥自己的作用。计算机本身扮演上述文明的敌手, 为全球资源与 技术进步展开竞争。文明的进程为玩家所左右, 游戏本身可以接纳不同类型的玩家, 从追求社 会和谐的理想主义者, 到好勇斗狠的功利主义者。上述进程或许可以理解为地球历史的写照, 但因拥有多种版本而与真正的历史相区别。人们可以在史前就建造铁路, 也可以变成战无不胜 的拿破仑。赢得胜利的标志, 是征服其他一切文明, 或者将 2 万人送入空间。默里认为: 当 然, 我们必须进一步分析上述游戏所包含的思想观念或价值判断。相比之下, 《西蒙城》的倾 向性是通过游戏当中的种种事变 (如社会动荡等) 相对间接地表现出来的, 玩家可能不知不觉 受其影响。《文明》则通过标题直截了当地告诉玩家说: 游戏所提供的是某个人对于人类历史 的解释, 而非科学的图式。游戏也提醒玩家: 每个领袖的行为是三种变量的结果, 一是侵犯性 与友好性, 二是扩张主义与完美主义, 三是穷兵黩武与文明立国。玩家可以接受它们, 也可以 对某种变量加以抑制。不同游戏的倾向性有隐显之别, 其价值标准并非都为人们所广泛接受。 就以后一种而论, 如何界定游戏的胜利, 便是一个有争议的问题。例如, 为什么一定是征服其 他文明才是胜利呢? 不同文明之间为何不能建立彼此合作、互惠互利的关系呢? 这种关系为何 不能作为胜利的标志? (Murray, Janet Horowitz. Hamlet on the Holodeck. New York, NY: The Free Press, 1997:89).

应当看到: 不论交互性叙事的创作或鉴赏, 都需要在实践中求得发展。在这一过程中, 原先持肯定或否定态度的人都有向对立的或中性的观点转变的可能。渊源有自的交互性叙事因 为 20 世纪新媒体革命的缘故而焕发青春, 从实践和理论两方面为叙事学的发展创造了契机。 处在这样的时代, 我们应当因时顺变, 深化叙事学的研究。 


\section{DAFTAR PUSTAKA}

Grodal, T. 2003. Stories for eye, ear, and muscles: video games, media, and embodied experiences. In The video game theory reader. Edited by Mark J. P. Wolf, and Bernard Perron. New York: Routledge.

Laurel, B. 1991. Computers as theatre. Reading, Mass.: Addison-Wesley Publishing Company.

Murray, J. H. 1997. Hamlet on the holodeck. New York, NY: The Free Press.

Ryan, Marie-Laure. 2001. Narrative as virtual reality: immersion and interactivity in literature and electronic media. Baltimore and London: The Johns Hopkins University Press.

Ryan, Marie-Laure. 2005. Peeling the onion: Layers of interactivity in digital narrative texts. Based on a talk presented at the Conference "Interactivity of Digital Texts," Münster, Germany, May 2005.

Sobral,.D., et al. 2003. Managing authoring in plot conduction. In Virtual Storytelling: Using virtual reality technologies for storytelling. Second International Conference, ICVS 2003, Proceedings. Edited by Olivier Balet, Gérard Subsol, and Patrice Torguet. Berlin: Springer.

Szilas, N. 2003. Authoring Highly Generative Interactive Drama. In Virtual Storytelling: Using Virtual Reality Technologies for Storytelling. Second International Conference. ICVS 2003, Proceedings. Edited by Olivier Balet, Gérard Subsol, and Patrice Torguet. Berlin: Springer. 